\title{
Analytical model of leakage through fault to overlying formations
}

GCCC Digital Publication Series \#12-08

\author{
Mehdi Zeidouni
}

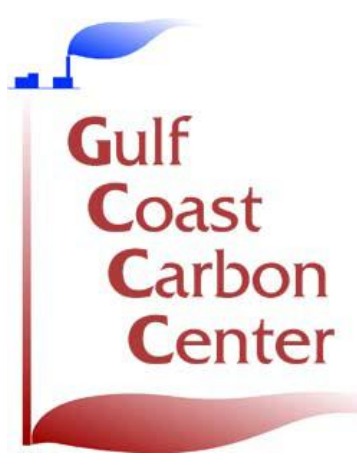

Keywords:

Modeling-Flow simulation, Monitoring-downhole pressure, Risk-fault, Riskenvironmental

Cited as:

Zeidouni, M., 2012, Analytical model of leakage through fault to overlying formations: Water Resour. Res., doi:10.1029/2012WR012582, (in press). GCCC Digital Publication Series \#12-08. 


\title{
Analytical Model of Leakage through Fault to Overlying Formations
}

Mehdi Zeidouni, Bureau of Economic Geology, The University of Texas at Austin, TX, USA

Phone: +1-512-471-4560, Fax: +1-512-471-0140, Email: mehdi.zeidouni@beg.utexas.edu

\begin{abstract}
A fault is generally composed of a fault core surrounded by damage zones and can accommodate both lateral and vertical flow. In this paper we develop an analytical model to evaluate the leakage rate through a fault and corresponding pressure changes in the injection zone and a shallower permeable interval. The leaky fault connects the upper interval and the target zone, which are otherwise separated by a confining layer. We account for both across-fault and upfault flow to honor the general architecture of the fault. We extend the two-formation analytical solution to consider multiple overlying formations with alternating confining layers offset by the fault. The solution methodology involves writing and transforming the coupled governing flow equations successively into the Laplace and Fourier domains and solving the resulting ordinary differential equations. The solution is verified through comparison with existing analytical solutions for bounding cases. Two examples are presented to demonstrate behavior and potential applications of our analytical model.
\end{abstract}

\section{Introduction}

The structure of a fault may be characterized by a fault core surrounded by damage zones (Figure 1). The lower permeability fault core generally includes a clay-rich gouge zone, where most displacement is accommodated. The surrounding damage zones generally involve fractures over a wide range of length scales characterized by enhanced permeability. The sealing capacity of 
the fault is controlled mainly by the lithologies juxtaposed on the walls of the fault and the conductivity contrast between the fault core and damage zones. Generally a fault may act as conduit, barrier, or combined conduit-barrier system that enhances or hinders fluid flow laterally [Caine et al., 1996; Anderson, 2006; Wibberley et al., 2008, and references therein; Faulkner et al., 2010]. The fault simultaneously can act as a pathway for vertical flow (leakage), especially when it is laterally sealing. In such a case, the fault core makes a strong barrier to horizontal fluid flow, whereas a higher permeability damage zone may form a preferential path to vertical flow. Bense et al. [2003] used hydraulic head data to illustrate that faults in the Roer Valley Rift system, Netherlands, form preferential paths to vertical groundwater flow while acting as a barrier to horizontal groundwater flow.

Normally separated permeable formations may be hydraulically connected by faults that allow for fluid interchange between them. Such fluid interchanges may be especially important in the case of strong and widespread pressure perturbations in the connected formations, which may be caused by production of oil, gas and water, or injection of fluids at high volume for waste disposal, gas storage, or geological storage of $\mathrm{CO}_{2}$ [Cihan et al., 2011]. Remedies such as brine extraction and reinjection into overlying aquifers and varying wells' configurations have been suggested to minimize these pressure perturbations [Ghaderi et al., 2009; Réveillère et al., 2012; Buscheck et al., 2012; Hosseini and Nicot, 2012]. The potential for leakage of $\mathrm{CO}_{2}$ and native fluids via fractures and faults is an area of considerable uncertainty for geological storage of $\mathrm{CO}_{2}$ [Benson and Cook, 2005; Nicot, 2008; Jordan et al., 2011; Stauffer et al., 2011; Selvadurai, 2012; Watson et al., 2012].

It is important to estimate the potential amount of leakage and corresponding pressure changes in formations connected through a vertically leaky fault. In this paper, we present a single-phase 
analytical model for fluid flow through a laterally and vertically leaky fault. The analytical model is used to evaluate the leakage rate and pressure perturbations related to fault leakage, both in the injection zone and in an overlying formation separated by a single confining layer.

Sedimentary basins commonly consist of multiple permeable formations with alternating lowpermeability or impermeable formations. Disposal of unwanted fluids generally takes place in a deep formation that may be overlain by multiple permeable formations with alternating confining layers. A leaky fault intersecting the target formation may offset multiple upper formations and extend to shallow depths. It is required to find that how the leakage from the injection zone will be attenuated toward the surface over the multiple layers intersecting the fault. This may have implications for the extent to which shallow aquifers may be contaminated by leakage from the injection zone. To answer questions related to such a multilayer system, the analytical solution developed for two connected zones is extended to enable evaluation of vertical leakage attenuation by considering multiple overlying formations with alternating confining layers. Such calculations can be done quickly without the need for spatial or time discretization, which can ease uncertainty quantification and Monte-Carlo-type analysis. The model can also be used to characterize a fault based on above-zone pressure monitoring [e.g., Sun and Nicot, 2012].

A method of images [Ferris, 1949; Jacob; 1950] may be the first analytical solution to transient flow in a system involving a fault. Bixel et al. [1963] obtained a solution to pressure diffusivity equation considering a planar interface in an infinite system. They assumed that pressure and flow rate are continuous at the planar discontinuity. The system was divided into two semiinfinite media with constant and distinct petrophysical properties (thickness, permeability, porosity, and compressibility) on each side of the planar interface, and fluid was injected (produced) at a constant rate at a well fully penetrating the reservoir. Yaxley [1987] presented a 
solution to Bixel et al.'s problem by considering a distinct transmissibility for the planar interface and, therefore, a pressure discontinuity. The same reservoir properties were used on sides of the fault. Ambastha et al. [1989] improved Yaxley’s solution by accounting for change in properties across the fault. Raghavan [2010] provided a new solution for the problem presented by Bixel et al. [1963] by making use of a combination of the Laplace transform and Green's function. These analytical models allow for lateral flow across the fault. Tangential flow parallel to the fault plane is also accounted for by considering pressure gradient along the fault plane. However, the effect of permeability contrast between the reservoir and the fault on tangential flow is neglected. Abbaszadeh and Cinco-ley [1995] accounted for tangential flow by considering along-fault conductivity and the transverse skin factor representing across-fault conductance. They used the Duhamel superposition integral as well as an image method to model pressure perturbations. Their solution involves complex “improper” integrals that are discretized and taken to the Laplace domain for numerical inversion. Anderson [2006] developed a steady-flow analytical solution that also accounts for tangential flow. He considered the fault an anisotropic medium with principal directions normal (x-direction) and tangential to the fault (y-direction). Rahman et al. [2003] modified Abbaszadeh and Cinco-ley’s solution by accounting for diffusivity inside the fault. They showed that transient effects within the fault may be important at an early time period.

Shan et al. [1995] developed an analytical model that allowed for vertical flow in the fault plane. The fault connects the target formation to an upper permeable zone from which it is separated by an impermeable confining layer. They presented solutions to evaluate leakage rate and pressure perturbations in the target zone and an upper formation. In development of their analytical model, however, Shan et al. [1995] neglected fault resistance to horizontal flow in the target 
formation. As a result, the pressure is considered laterally continuous across the fault. However, the fault may act as a barrier to lateral flow, given a well-developed fault core that leads to lateral pressure discontinuity across the fault. Our approach in modeling fault leakage is similar to that presented by Shan et al. [1995]. However, unlike them, we account for pressure discontinuity across the fault that leads to different leakage rates and pressure perturbations on two sides of the fault in the upper formation. Next, we extend the two-layer solution to consider multiple overlying permeable formations intersecting the fault. Whereas the analytical solution presented here can be applied to both production and injection, we consider injection throughout the derivations for consistency. We consider injection in a target formation and present the solution in terms of leakage rate and pressure perturbations in the interconnected formations.

The paper is organized as follows. Section 2 presents problem description, definitions, and assumptions. Flow equations, along with initial and boundary conditions for a two-layer system, are presented and solved in Section 3. Determining valid assumptions to simplify the solution, we extend the model to a system of $N$ overlying permeable formations with alternating confining layers intersected by a leaky fault in Section 4. Section 5 includes example calculations to (1) validate the new solution by comparison to existing analytical solutions, (2) analyze its behavior, and (3) demonstrate its application.

\section{Problem description and assumptions}

The simplified configuration modeled herein (Figure 2) is an idealization of the system shown in Figure 1, in which the fault is considered a vertical, linear, semipermeable interface and the vertical displacement is neglected. Although this is a simplification of the complex nature of the fault zone, it has the essential property of imposing a linear flow pattern at the fault plane 
[Stewart et al., 1984]. Fault storativity is neglected, which may be valid when the fault thickness is smaller than the fault distance from the well and/or when the fault zone porosity and compressibility are small. We neglect the effect of contrast between along-fault and reservoir permeabilities on the tangential flow parallel to the fault plane. This assumption may be valid because the fault damage zone is highly heterogeneous and may not be well connected along the fault. As a result of neglecting fault-storativity and the tangential-flow induced by permeability contrast between the reservoir and the fault, what enters the fault can either leak to the upper formation or advance to the other side of the fault.

Further, we assume that (1) the system is bounded from top and bottom by no-flow boundary condition; (2) initially the system is in hydrostatic equilibrium, which is then perturbed by a constant-injection-rate well that fully penetrates the injection zone; (3) the formations are homogeneous and isotropic with uniform thickness; (4) the injection well fully penetrates the injection zone and can be approximated as a line sink; (5) reservoir and injection fluids are the same—-single phase and slightly compressible with constant compressibility and viscosity; (6) the lateral flow rate crossing the fault is proportional to the instantaneous pressure difference between the two sides of the fault; (7) lateral communication across the fault in the confining layer is negligible; and (8) the fault extends infinitely and divides the model domain into two semi-infinite media: region 1 is the right side of the fault where the well is located, and region 2 is the left side. The Cartesian coordinate is set such that the fault plane lies along the $y$-axis and the $\mathrm{x}$-axis goes through the injection well perpendicular to the fault plane.

\section{Analytical model}


In modeling the fault system (Figure 2), we closely follow the model developed by Shan et al. [1995]. However, unlike their model, we account for fault resistance to lateral flow. By accounting for lateral flow the inter-formational vertical flow is also affected. Leakage rate on the two sides of the fault plane is no longer the same.

To properly model the fault, there may be two approaches. One approach is to lump fault core and its surrounding damage zones into a single zone with distinct vertical and horizontal permeabilities (see Figure 3a). Such an approach will make the vertically leaked fluids equally available to both sides of the fault in the upper formation. However, leakage to either side of the fault in the upper interval may not be equal. If the ratio of the fault's vertical transmissibility to its lateral transmissibility is large, most leakage should flow to the side of the fault corresponding to the injection well.

The second approach is to let the vertical component of flow occur only through damage zones on both sides of the fault (see Figure 3b). In such an approach, the leakage rate will not be bisected (divided into two identical parts) to the sides of the fault while the two sides are in communication, given fault lateral transmissibility. This approach is more consistent with the commonly described fault structure and is considered in this paper. We assign a distinct horizontal permeability to the fault, and the two sides of the fault are assigned distinct and equal vertical permeabilities so as to simulate vertical flow through the damage zones. It is doubtful that differences in vertical permeability of the damage zones may ever be known.

Governing equations and corresponding boundary conditions are set up separately for each region of each interval. For the injection zone, we can write a special form of the diffusivity equation for regions 1 and 2, respectively: 
$\frac{\partial^{2} \Delta P_{1}}{\partial x^{2}}+\frac{\partial^{2} \Delta P_{1}}{\partial y^{2}}+\frac{q \mu}{k h} \delta(x-a) \delta(y)=\frac{1}{\eta} \frac{\partial \Delta P_{1}}{\partial t}$

$\frac{\partial^{2} \Delta P_{2}}{\partial x^{2}}+\frac{\partial^{2} \Delta P_{2}}{\partial y^{2}}=\frac{1}{\eta} \frac{\partial \Delta P_{2}}{\partial t}$

where $\delta$ is the Dirac delta function, $q$ is volumetric injection rate, $\mu$ is fluid viscosity, and $k, h$, and $\eta$ are injection-zone permeability, thickness, and diffusivity coefficient respectively; $a$ is the distance of the injection well to the fault plane. The diffusivity coefficient, $\eta$, is defined as $k /(\phi \mu$ $c_{t}$ ) where $\phi$ and $c_{t}$ are porosity and total compressibility respectively. $\Delta P_{1}$ and $\Delta P_{2}$ stand for pressure changes in regions 1 and 2, respectively, and are defined as $\Delta P_{j}=P_{0}-P_{j}$, where $j=1,2$ and $P_{0}$ is the initial pressure of the injection zone. Corresponding initial and boundary conditions are given by:

$$
\begin{aligned}
& \Delta P_{1}(x, y, 0)=\Delta P_{2}(x, y, 0)=0 \\
& \Delta P_{1}(x, \pm \infty, t)=\Delta P_{2}(x, \pm \infty, t)=0 \\
& \Delta P_{1}(+\infty, y, t)=\Delta P_{2}(-\infty, y, t)=0 \\
& \frac{k h}{\mu} \frac{\partial \Delta P_{1}(0, y, t)}{\partial x}=\frac{k_{f h} h}{\mu w_{f}}\left(\Delta P_{1}(0, y, t)-\Delta P_{2}(0, y, t)\right)+\frac{k_{f v} w_{f}}{2 \mu L}\left(\Delta P_{1}(0, y, t)-\Delta P_{u 1}(0, y, t)\right) \\
& \frac{k h}{\mu} \frac{\partial \Delta P_{2}(0, y, t)}{\partial x}+\frac{k_{f v} w_{f}}{2 \mu L}\left(\Delta P_{2}(0, y, t)-\Delta P_{u 2}(0, y, t)\right)=\frac{k_{f h} h}{\mu w_{f}}\left(\Delta P_{1}(0, y, t)-\Delta P_{2}(0, y, t)\right)
\end{aligned}
$$

where $k_{f h}$ and $w_{f}$ are effective horizontal permeability and effective width of the fault zone, respectively [Stewart et al., 1984; Yaxley, 1987]; $k_{f v}$ is the effective vertical permeability of the damage zones; and $L$ is the leakage interval. Fault's lateral and vertical transmissibilities are given by $k_{f h} . h$ and $k_{f v} \cdot w_{f} / 2$ respectively. The initial condition given by Equation (3) simply means that pressure is initially the same for all $x$ and $y$ in the formation. Equations (4) and (5) ensure transient-flow conditions, considering negligible pressure change at the outer infinite boundaries. 
Equation (6) states that the fault outflow at region 1 is the sum of the flow toward region 2 and the (vertical) leakage rate at the side of region 1. Equation (7) presents the relationship between flow rate entering region 2, pressure difference on two sides of the fault, and leakage to region 2 of the upper formation.

Similarly for the upper zone we can write:

$$
\begin{aligned}
& \frac{\partial^{2} \Delta P_{u 1}}{\partial x^{2}}+\frac{\partial^{2} \Delta P_{u 1}}{\partial y^{2}}=\frac{1}{\eta_{u}} \frac{\partial \Delta P_{u 1}}{\partial t} \\
& \frac{\partial^{2} \Delta P_{u 2}}{\partial x^{2}}+\frac{\partial^{2} \Delta P_{u 2}}{\partial y^{2}}=\frac{1}{\eta_{u}} \frac{\partial \Delta P_{u 2}}{\partial t}
\end{aligned}
$$

where subscript $u$ stands for the upper zone. $\Delta P_{u j}=P_{u 0}-P_{u j}$ for $j=1,2$ and $P_{u 0}$ is the initial pressure of the upper zone. Initial and boundary conditions are given by:

$$
\begin{aligned}
& \Delta P_{u 1}(x, y, 0)=\Delta P_{u 2}(x, y, 0)=0 \\
& \Delta P_{u 1}(x, \pm \infty, t)=\Delta P_{u 2}(x, \pm \infty, t)=0 \\
& \Delta P_{u 1}(+\infty, y, t)=\Delta P_{u 2}(-\infty, y, t)=0 \\
& \frac{k_{f v} w_{f}}{2 \mu L}\left(\Delta P_{1}(0, y, t)-\Delta P_{u 1}(0, y, t)\right)=\frac{k_{f h} h_{u}}{\mu w_{f}}\left(\Delta P_{u 1}(0, y, t)-\Delta P_{u 2}(0, y, t)\right)-\frac{k_{u} h_{u}}{\mu} \frac{\partial \Delta P_{u 1}(0, y, t)}{\partial x} \\
& \frac{k_{f v} w_{f}}{2 \mu L}\left(\Delta P_{2}(0, y, t)-\Delta P_{u 2}(0, y, t)\right)+\frac{k_{f h} h_{u}}{\mu w_{f}}\left(\Delta P_{u 1}(0, y, t)-\Delta P_{u 2}(0, y, t)\right)=\frac{k_{u} h_{u}}{\mu} \frac{\partial \Delta P_{u 2}(0, y, t)}{\partial x}
\end{aligned}
$$

Equation (13) asserts that leakage from region 1 is the sum of the inflow to region 1 of the upper zone and across-fault flow in the upper zone. Equation (14) relates leakage rate at region 2 to the across-fault flow in the upper zone and the inflow of region 2 of the upper zone.

The system is reduced to a system of coupled ODEs using the Laplace transform in time and the exponential Fourier transform in $y$-coordinate, defined by: 


$$
\begin{aligned}
& \overline{\Delta P}(x, y, s)=\mathcal{L}\{\Delta P(x, y, t)\}=\int_{0}^{\infty} \Delta P(x, y, t) e^{-s t} d t \\
& \overline{\overline{\Delta P}}(x, \omega, s)=\mathcal{F}\{\overline{\Delta P}(x, y, s)\}=\int_{-\infty}^{\infty} \overline{\Delta P}(x, y, s) e^{i \omega y} d y
\end{aligned}
$$

Details of the solution for the above coupled system of PDE's are given in Appendix A. The following is obtained for the dimensionless pressure change in regions 1 and 2 of the injection and upper zones:

$$
\begin{aligned}
& \overline{\bar{P}}_{D 1}=\frac{1}{2 s A}\left(e^{-A A x_{D}-11}+\frac{A^{2}\left(\alpha_{u}+A_{u} T_{D}\right)\left(\alpha_{u}+A_{u} T_{D}+2 \alpha h_{D}\right)-2 \alpha \alpha_{u} A_{u} T_{D}\left(\alpha_{u}+2 \alpha h_{D}+\alpha_{u} h_{D}+A_{u} T_{D}\right)-\alpha_{u} A_{u} T_{D} \alpha_{u}}{\left(A a_{u}+A A_{u} T_{D}+a_{u} A_{u} T_{D}\right)\left(2 a a_{u}+A a_{u}+4 a^{2} h_{D}+2 a A h_{D}+2 a a_{u} h_{D}+2 a A_{u} T_{D}+A A_{u} T_{D}+a_{u} A_{u} T_{D}\right)} e^{-A\left(x_{D}+1\right)}\right) \\
& \overline{\bar{P}}_{D 2}=\frac{\alpha}{s}\left(\frac{\alpha_{u}\left(\alpha_{u}+2 \alpha h_{D}+\alpha_{u} h_{D}\right)+2 A_{u} T_{D}\left(\alpha_{u}+\alpha h_{D}\right)+A_{u}^{2} T_{D}^{2}}{\left(A a_{u}+A A_{u} T_{D}+a_{u} A_{u} T_{D}\right)\left(2 a a_{u}+A a_{u}+4 a^{2} h_{D}+2 a A h_{D}+2 a a_{u} h_{D}+2 a A_{u} T_{D}+A A_{u} T_{D}+a_{u} A_{u} T_{D}\right)} e^{A\left(x_{D}-1\right)}\right) \\
& \overline{\bar{P}}_{D u 1}=\frac{\alpha_{u}}{s}\left(\frac{\alpha \alpha_{u}+A \alpha_{u}+2 \alpha^{2} h_{D}+\alpha A h_{D}+\alpha \alpha_{u} h_{D}+\alpha A_{u} T_{D}+A A_{u} T_{D}+\alpha_{u} A_{u} T_{D}}{\left(A a_{u}+A A_{u} T_{D}+a_{u} A_{u} T_{D}\right)\left(2 a a_{u}+A a_{u}+4 a^{2} h_{D}+2 a A h_{D}+2 a a_{u} h_{D}+2 a A_{u} T_{D}+A A_{u} T_{D}+a_{u} A_{u} T_{D}\right)} e^{-A_{u} x_{D}-A}\right) \\
& \overline{\bar{P}}_{D u 2}=\frac{\alpha \alpha_{u}}{s}\left(\frac{\alpha_{u}+2 \alpha h_{D}+A h_{D}+\alpha_{u} h_{D}+A_{u} T_{D}}{\left(A a_{u}+A A_{u} T_{D}+a_{u} A_{u} T_{D}\right)\left(2 a a_{u}+A a_{u}+4 a^{2} h_{D}+2 a A h_{D}+2 a a_{u} h_{D}+2 a A_{u} T_{D}+A A_{u} T_{D}+a_{u} A_{u} T_{D}\right)} e^{A_{u} x_{D}-A}\right)
\end{aligned}
$$

where

$$
\begin{aligned}
& P_{D 1}=\frac{k h}{q \mu} \Delta P_{1}, P_{D 2}=\frac{k h}{q \mu} \Delta P_{2}, P_{D u 1}=\frac{k h}{q \mu} \Delta P_{u 1}, P_{D u 2}=\frac{k h}{q \mu} \Delta P_{u 2}, t_{D}=\frac{\eta t}{a^{2}}, x_{D}=\frac{x}{a}, y_{D}=\frac{y}{a} \\
& \alpha=\left(\frac{k_{f h}}{w_{f}}\right) /\left(\frac{k}{a}\right), \alpha_{u}=\left(\frac{k_{f v} w_{f}}{2 L}\right) /\left(\frac{k h}{a}\right), h_{D}=\frac{h_{u}}{h}, T_{D}=\frac{k_{u} h_{u}}{k h}, \eta_{D}=\frac{\eta_{u}}{\eta} \\
& A^{2}=\omega^{2}+s, A_{u}^{2}=\omega^{2}+\frac{s}{\eta_{D}}
\end{aligned}
$$

The "=” sign on top of dimensionless pressure represents the transformed dimensionless pressure into the Laplace and Fourier domains. As a result of considering the fault's lateral resistance to flow, two new dimensionless terms appear in the solution: $\alpha$, which is a measure of fault lateral 
transmissibility, and $h_{D}$, which is the ratio of upper-zone thickness to that of the injection zone. When $\alpha=0$ the fault is horizontally sealing and hinders the pressure communication between two sides of the fault. When $\alpha \rightarrow \infty$ the fault is fully conductive in $x$-direction, the pressure is identical at both sides at the fault plane, and our solution reduces to that given by Shan et al. [1995]. Analytical inversion of the above solution to time and space domain is difficult; the inverse is obtained numerically as described in Appendix B. Whereas the injection rate is taken as a constant in the above model, the solution can be applied to a variable-rate problem using the superposition principle. Note that different vertical permeabilities can be assigned to the damage zones. As a result, one more dimensionless group will appear which further complicates the solution. In such an event, we obtain $\alpha_{u 1}$ and $\alpha_{u 2}$ (for damages zones at the sides of regions 1 and 2 respectively) rather than a single $\alpha_{u}$.

We can now use the pressure difference between the injection zone and the upper formation at the fault plane to obtain the leakage rate to the upper formation. Leakage flux at an arbitrary value of $y$ along the fault plane $(x=0)$ is given by:

leakage flux $=\frac{k_{f v} w_{f}}{2 \mu L}\left(\left(\Delta P_{1}(0, y, t)-\Delta P_{u 1}(0, y, t)\right)+\left(\Delta P_{2}(0, y, t)-\Delta P_{u 2}(0, y, t)\right)\right)$

Integrating the flux over $y$ from $-\infty$ to $+\infty$, the total leakage rate to the upper formation through the fault can be obtained by [Shan et al., 1995]:

$q_{l}=\frac{k_{f v} w_{f}}{2 \mu L}\left(\int_{y=-\infty}^{\infty}\left(\Delta P_{1}(0, y, t)-\Delta P_{u 1}(0, y, t)\right) d y+\int_{y=-\infty}^{\infty}\left(\Delta P_{2}(0, y, t)-\Delta P_{u 2}(0, y, t)\right) d y\right)$

which in dimensionless form can be written as:

$$
q_{I D}=\alpha_{u}\left(\int_{y_{D}=-\infty}^{\infty}\left(P_{D 1}\left(0, y_{D}, t_{D}\right)-P_{D u 1}\left(0, y_{D}, t_{D}\right)\right) d y_{D}+\int_{y_{D}=-\infty}^{\infty}\left(P_{D 2}\left(0, y_{D}, t_{D}\right)-P_{D u 2}\left(0, y_{D}, t_{D}\right)\right) d y_{D}\right)
$$


where $q_{l D}\left(=q_{l} / q\right)$ is the dimensionless leakage rate through the fault. Combining Equation (26) with dimensionless form of Equations (13) and (14) gives:

$$
q_{I D}=T_{D} \int_{y_{D}=-\infty}^{\infty}\left(\frac{\partial P_{D u 2}\left(0, y_{D}, t_{D}\right)}{\partial x_{D}}-\frac{\partial P_{D u 1}\left(0, y_{D}, t_{D}\right)}{\partial x_{D}}\right) d y_{D}
$$

Evaluating the integrand in the Laplace-Fourier domain, we obtain:

$$
\left(\frac{d \overline{\bar{P}}_{D u 2}(0, \omega, s)}{d x_{D}}-\frac{d \overline{\bar{P}}_{D u 1}(0, \omega, s)}{d x_{D}}\right)=\frac{A_{u} \alpha_{u}}{A \alpha_{u}+A A_{u} T_{D}+\alpha_{u} A_{u} T_{D}} \frac{e^{-A}}{s}
$$

This equation clearly shows that the leakage rate is independent of parameters $\alpha$ and $h_{D}$, which are dimensionless groups identified as a result of including the resistance of the fault to lateral flow. In other words, assuming perfect horizontal communication will not affect the total leakage rate to the upper interval. Therefore, the leakage rate evaluated by Shan et al. [1995] would be identical to that obtained by the solution presented here. Thus, for mere calculation of leakage rate, the lateral resistance of the fault plane can be neglected. Independence of the leakage rate from the parameters $\alpha$ and $h_{D}$ implies that the arithmetic mean of the pressure change at the fault plane will also be independent of $\alpha$ and $h_{D}$. This is demonstrated analytically in Appendix A by equations (A45) and (A46).

\section{Extension to multiple upper formations and confining layers}

In this section, we extend the solution derived for the two-layer system to consider $N$ overlying formations separated by confining layers. In such a system, obtaining the leakage rate is more relevant than accessing the pressure attenuation caused by leak-off into the upper formations. Focusing on the leakage rate allows the model developed earlier to be further simplified. As shown earlier, the leakage rate is independent of fault horizontal permeability. Therefore, we 
extend the Shan et al. [1995] solution (which is a limiting case of our model for $\alpha \rightarrow \infty$ ), considering that pressure symmetrically rises around the fault plane at the upper formations. Therefore, $\Delta P_{u 1, i}=\Delta P_{u 2, i}$ for $i=1,2, \ldots, N$, where $N$ is number of overlying formations. Details of the analytical solution for the multilayer system (Figure 4) are given in Appendix C. As shown in Figure $4, i=1$ for the formation immediately above the injection zone and $i=N$ for the uppermost (shallowest) interval. The leakage rate to the $i^{\text {th }}$ interval is given by:

$q_{l D i}=2 T_{D i} \int_{-\infty}^{\infty} \frac{\partial P_{D u 2, i}\left(0, y_{D}, t_{D}\right)}{\partial x_{D}} d y_{D}$ for $i=1,2, \ldots, N$

where $T_{D i}$ and $P_{D u 2, i}$ are dimensionless transmissivity and dimensionless pressure, respectively, in region 2 of the $i$ 'th formation. Dimensionless pressure should be evaluated by inversion from the Laplace-Fourier domain and is given by:

$\overline{\bar{P}}_{D u 2, i}=C_{u i} e^{A_{u i} x_{D}}$ for $i=1,2, \ldots, N$

the derivative of which, with respect to $x_{D}$ at $x_{D}=0$, is:

$$
\left.\frac{d \overline{\bar{P}}_{D u 2, i}}{d x_{D}}\right|_{x_{D}=0}=C_{u i} A_{u i} \text { for } i=1,2, \ldots, N
$$

for which $A_{u i}^{2}=\omega^{2}+\frac{s}{\eta_{D i}}$.

Unlike the two-layer system solution, it is not easy to derive the coefficients explicitly. The coefficients are determined by solving the following system of linear algebraic equations:

H . $C=F$

where

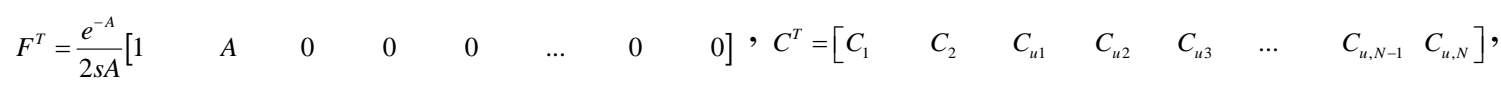

and 
$H=\left[\begin{array}{llllllll}-1 & 1 & 0 & 0 & 0 & \ldots & 0 & 0 \\ A & A+2 \alpha_{u 1} & -2 \alpha_{u 1} & 0 & 0 & \ldots & 0 & 0 \\ 0 & -\alpha_{u 1} & \alpha_{u 1}+T_{D 1} A_{u 1} & T_{D 2} A_{u 2} & T_{D 3} A_{u 3} & \ldots & T_{D, N-1} A_{u, N-1} & T_{D, N} A_{u, N} \\ 0 & 0 & -\alpha_{u 2} & \alpha_{u 2}+T_{D 2} A_{u 2} & T_{D 3} A_{u 3} & \ldots & T_{D, N-1} A_{u, N-1} & T_{D, N} A_{u, N} \\ 0 & 0 & 0 & -\alpha_{u 3} & \alpha_{u 3}+T_{D 3} A_{u 3} & \ldots & T_{D, N-1} A_{u, N-1} & T_{D, N} A_{u, N} \\ . & & & & & & \\ . & & & & & & & \\ . & 0 & 0 & 0 & 0 & \ldots & \alpha_{u, N-1}+T_{D, N-1} A_{u, N-1} & T_{D, N} A_{u, N} \\ 0 & 0 & 0 & 0 & 0 & \ldots & -\alpha_{u, N} & \alpha_{u, N}+T_{D, N} A_{u, N}\end{array}\right]$

Superscript $T$ indicates transpose of the vector. The matrix $\boldsymbol{H}$-the size of which is $(\mathrm{N}+2) \times$ $(\mathrm{N}+2)$ - is inverted by standard techniques and multiplied by vector $F$ to determine the coefficients, $C_{u i}$. The pressure derivative is then evaluated, the integration of which (based on Equation (29)) provides the leakage rate to $i^{\text {th }}$ overlying formation. In fact, the above matrix approach can be used for the two-layer system, instead of the solution being presented in a complicated, explicit form, as given by Equations (17) through (20). In addition, to account for the effect of lateral resistance of the fault in the multilayer system, the number of coefficients (length of vector $C$ ) will be $2 N+2$. The size of the corresponding $\boldsymbol{H}$ matrix will be $(2 N+2) \times$ $(2 N+2)$, which complicates the solution procedure without adding information on leakage rates to each interval. Derivation of the two-layer leakage rate based on the above matrix-based approach for the multilayer system is given Appendix D.

\section{Example calculations}

To validate our solution and show its behavior and potential applications, we present solutions for two examples. The first example is a two-layer system earlier solved by Shan et al. [1995] considering zero and nonzero resistance to flow in an upper aquifer. This example is used to validate our solution and find the effect of newly identified dimensionless groups when considering the fault's lateral resistance to flow. The second example investigates the effect of 
alternating permeable formations and confining layers on up-fault leakage. To demonstrate the effect of such a multilayer system on leakage attenuation, we compare the solution with a twolayer system comprising the same injection zone and an overlying formation and intervening confining layer with equal thickness to the multilayer system. The calculations are performed by programming the analytical solutions in MATLAB.

\subsection{Example 1: Comparison with solutions of Shan et al. [1995]}

The parameters for this example are taken from Shan et al. [1995], and the system involves two aquifers separated by an impermeable layer. They present their solutions considering zero and non-zero resistance to flow from the upper aquifer. They consider $T_{D}=1$ and $\alpha_{u}=0.02$ for the nonzero resistance case. In Figure 5, the dimensionless pressure for this case is plotted versus time and compared with results presented in Figure 5 of Shan et al. [1995]. Pressure is given at the injection zone halfway between the injection well and the fault $\left(x_{D}=0.5\right)$. In Shan et al., the fault's horizontal resistance to flow is neglected $(\alpha \rightarrow \infty)$. Therefore, the solutions match for $\alpha \rightarrow \infty$ (the actual number used here is $\alpha=10^{9}$ ), and as $\alpha$ decreases pressure increases as well. However, for the early time period, solutions for various values of $\alpha$ are the same.

Shan et al. [1995] also present dimensional results for a case considering negligible resistance to flow from the upper aquifer. For this example water is injected at a constant rate of $0.005 \mathrm{~m}^{3} / \mathrm{s}$ into the $20 \mathrm{~m}$ thick lower aquifer. The diffusivity coefficient $(\eta)$ for both the target and upper aquifers is $10 \mathrm{~m}^{2} / \mathrm{s}$, and the fault is $100 \mathrm{~m}$ from the injection well $(a=100 \mathrm{~m})$. The vertical leakage interval is $L=50 \mathrm{~m}$, the permeability of the lower aquifer is $10 \mathrm{D}$, and water viscosity is 1 cp. In Figure 6, the leakage rate to the upper interval is plotted versus dimensionless time and compared with results presented by Shan et al solution. A good agreement is achieved for all 
values of parameter $\alpha_{u}$. Increasing $\alpha_{u}$ leads to increased leakage rate and earlier advent of steady-state condition for which $q_{I D}=1$. Negligible resistance to flow in the upper aquifer can be achieved using our solution by considering $T_{D} \rightarrow \infty$ (the actual number used here is $T_{D}=10^{9}$ ).

Shan et al. also present head changes for the negligible resistance case considering $\alpha_{u}=1$. Results of our model are compared to those of Shan et al. in Figure 7 where the pressure change at the target aquifer after 10 hours of injection is evaluated along the $x-z$ plane. Head changes at the target aquifer [Shan et al., 1995, their Figure 2] are converted to pressure changes and plotted versus $x$ at the symmetry plane $(y=0)$. Good agreement between the solutions is observed. As mentioned earlier, pressure at the fault is continuous because the fault's resistance to lateral flow has been neglected, and pressure discontinuity at the fault plane is introduced by considering a finite $\alpha$ parameter. As a result, pressure change increases on the side of the injection well (region 1). Pressure change drops from 3.4 to $1.3 \mathrm{kPa}$ at the fault plane for $\alpha=1$. When $\alpha$ is reduced to zero, the fault becomes an absolute barrier to lateral flow, and the pressure change in region 2 will be zero. Note that the arithmetic mean of pressure change at the fault plane is constant as noted in Section 3. Figure 8 presents the pressure changes in the target and upper aquifers considering that the transmissivity of the upper aquifer is identical to that of the target aquifer $\left(T_{D}=1\right)$. Pressure change in the upper aquifer is no longer zero, forming resistance to up-fault leakage. As a result, pressure change in the target aquifer will be larger than that shown in Figure 7. Considering $\alpha=1$, pressure change in region 1 of the target aquifer at the fault plane is $6.2 \mathrm{kPa}$ when the upper aquifer shows resistance to flow $\left(T_{D}=1\right)$, compared with $3.4 \mathrm{kPa}$ when the upper aquifer's resistance to flow is neglected $\left(T_{D} \rightarrow \infty\right)$. Pressure in the upper aquifer is asymmetric (except for $\alpha \rightarrow \infty$ ) owing to higher rates into region 1 of the upper aquifer. The pressure change in region 1 of the upper aquifer is $0.6 \mathrm{kPa}$ larger than that in region 2 at the fault plane for $\alpha=1$. 
Dimensionless thickness, $h_{D}$, is another dimensionless term that is identified when fault resistance to lateral flow is included. The effect of varying $h_{D}$ on pressure is shown in Figure 9 considering $\alpha=1$. Pressure in region 1 increases and that in region 2 decreases as a result of decreasing $h_{D}$ (everything else being equal). Pressure in the upper zone is more sensitive to $h_{D}$ than it is in the target aquifer. For instance, pressure at the fault in region 1 increases by $2 \%$ as a result of decreasing $h_{D}$ from 100 to 1 , whereas the corresponding change for the upper aquifer is 8\%. As noted in Section 3 and shown by Equation (A46), the arithmetic mean of the pressure at the fault plane is the same for all values of $h_{D}$. Varying $h_{D}$ does not affect the solution for $\alpha=0$ or $\alpha \rightarrow \infty$. For $\alpha \rightarrow \infty$, leakage will be equally divided between regions 1 and 2 of the upper aquifer. For $\alpha=0$, because leakage rate can only enter region $1, h_{D}$ will have no effect on pressure in these cases.

\subsection{Example 2: Multilayer system}

For example 2, water is injected into a $60 \mathrm{~m}$ thick injection zone which is overlain by 15 aquifers separated by confining layers. Although our analytical solution can handle distinct properties for each layer, we consider properties of the overlying aquifers identical for convenience. All upper aquifers are $40 \mathrm{~m}$ thick, with $30 \mathrm{~m}$ thick intervening confining layers. Permeability is $100 \mathrm{mD}$, and the diffusivity coefficient $(\eta)$ is $10 \mathrm{~m}^{2} / \mathrm{s}$ for all layers, including the injection zone. The fault is $500 \mathrm{~m}$ from the injection well, intersecting all formations. Vertical transmissivity of the fault $\left(k_{f} \times w_{f}\right)$ is the same for all layers and equal to $1.68 \times 10^{-14} \mathrm{~m}^{3}\left(\alpha_{u, i}=0.01\right.$ for all $i$ 's except for $i=1$, for which $\left.\alpha_{u 1}=0.00875\right)$. Dimensionless leakage rate $\left(q_{I D}\right)$ and dimensionless leakage volume $\left(Q_{I D}\right)$ are plotted versus time for 100 years of injection (Figure 10a). $Q_{I D}$ is defined as the cumulative leakage volume divided by cumulative injected volume. Results are shown for three 
aquifers: the bottom aquifer (immediately above the injection zone, \#1), the middle aquifer (\#8), and the top aquifer (\#15), which is the shallowest aquifer intersected by the fault. At early time period, the leakage rate to the bottom layer is about 30 orders of magnitudes larger than the leakage rate to the shallowest aquifer. After 25 years of injection, this reduces to 4 orders of magnitudes. The dimensionless leakage volume to the top aquifer after 25 years of injection is about $10^{-5}$ (or 0.001 percent) of the total injected volume, whereas that to the bottom aquifer is about $20 \%$ of the total injected volume by this time.

To further investigate the behavior of fault leakage, Figure 10a is re-plotted in semi-logarithmic form and shown in Figure 10b. It is clearly visible from Figure 10b that the dimensionless leakage rate for the bottom interval decreases after initial increase. This is due to the increasing pressure in this interval which decreases the leakage driving force. As a result of reduced pressure difference between the injection zone and bottom aquifer, the leakage rate starts decreasing after 18 years of injection. The leakage rate will ultimately reach steady-state conditions when the total leakage rate equals the injection rate at the injection zone. Due to identical properties of all overlying aquifers, the steady-state leakage rate will be equally distributed over all formations. Therefore, the dimensionless leakage rate for all overlying aquifers will ultimately $(t \rightarrow \infty)$ converge to the same value. This value is the ratio of the transmissivity of a given overlying aquifer to the total transmissivity of overlying aquifers and the injection zone i.e. $0.061(=40 /(15 \times 40+60))$.

Results of this example multilayer system are compared with those of an equivalent two-layer system in Figures 10 and 11 similar to the analysis of leaky wells presented by Nordbotten et al. [2004]. For the equivalent two-layer system, thickness of the upper aquifer is the sum of all overlying aquifers $(600 \mathrm{~m})$, and thickness of the confining layer is the sum of all alternating 
confining layers $(450 \mathrm{~m})$. For this two-layer system, $L=780 \mathrm{~m}, T_{D}=10$, and $\alpha_{u}=8.97 \times 10^{-4}$. Both dimensionless leakage rate and volume monotonically increase with time. Based on Figure 10, the leakage rate after 25 years reaches $15 \%$ of the injection rate and the total leakage volume reaches $11 \%$ of the total injection. By this time, the bottom aquifer (of the multilayer system) alone contributes to larger cumulative leakage than the equivalent, thick, overlying aquifer. This demonstrates the importance of the intervening layers in hindering up-fault leakage to shallow aquifers. The dimensionless leakage volume to the overlying formations after 1, 5, and 25 years of injection is plotted and compared with the leakage volume to the equivalent two-layer system (Figure 11). Although the attenuation of leakage caused by deep aquifers reduces over time, a large contrast between the leakages to deep versus shallow aquifers is nonetheless observed.

\section{Summary and Conclusions}

An analytical model is developed to evaluate leakage rates and pressure perturbations related to flow between formations linked by a leaky fault. The model is built on the basis of earlier work by Shan et al. [1995] on vertical communication between aquifers through a leaky fault. We made two modifications to their solution by (1) considering the fault resistance to lateral flow and (2) extending the model domain from a single, overlying formation to multiple overlying formations with alternating confining layers intersected by the fault. To honor lateral flow resistance, we assigned a finite and distinct lateral transmissibility to the fault. The system domain is divided into two regions at each side of the fault plane. Governing equations for each region of the injection zone and upper formation in the linked formations are written that are coupled through the boundary conditions written at the fault. The boundary conditions at the fault plane are established considering the general fault architecture being composed of a fault 
core surrounded by damage zones. The effect of damage zones to up-fault flow is modeled by allowing for two vertical pathways at the sides of the fault communicating horizontally based on horizontal transmissibility of the fault. To solve the resulting system, we take the coupled PDE's through Laplace transform followed by the exponential Fourier transform. The solution for pressure changes in each region of each formation is obtained in the Laplace-Fourier domain and is numerically inverted to achieve the solution in the time and space domain. The leakage rate through the fault is then obtained based on integration of the pressure difference between the linked formations along the fault. It is found that the leakage rate is independent of the horizontal transmissibility of the fault. This observation is next used to simplify obtaining the solution for a multi-formation system. The solution for a system of multiple overlying formations involves solving a system of linear algebraic equations to obtain pressure changes in the Laplace-Fourier domain. The pressure changes are then inverted and integrated to obtain the leakage rate. The solution procedures are programmed in MATLAB and can be obtained from the author upon request. The limitations of our analytical solutions are determined based on the assumptions made in their derivations.

The solution is validated by comparing results of an example problem presented by Shan et al. [1995]. It is shown that in consequence of considering the lateral resistance of the fault to flow, pressure discontinuity at the fault plane is introduced, and pressure change in the upper formation is no longer symmetric. The arithmetic mean of the pressure change at the fault plane is independent of parameter $\alpha$ (representing fault lateral resistance to flow) due to independency of the up-fault leakage rate from $\alpha$. We show that the ratio of the formations' thicknesses can impact the solution if $\alpha$ is a finite, nonzero value. 
Applying the solution to a multilayer system example, we next investigate the behavior of the leakage in the presence of more than a single overlying formation. It is found that the leakage rate may not monotonically increase due to excessive pressure increase in the upper formations that may increase resistance to flow and reduce the leakage rate over time. Thus, the contribution of bottom layers to attenuation of upward leakage may reduce over time. For a multilayer system, cumulative leakage to the top (shallowest) aquifer may be several orders of magnitude smaller than that to the bottom aquifer. If the multiple overlying aquifers and confining layers are replaced by a single, thick, overlying aquifer and confining layer, cumulative leakage to the single, thick aquifer may be even smaller than that to the bottom aquifer of the multilayer system. These observations demonstrate the effectiveness of a multiple-layer system in attenuation and reduction of leakage to shallow aquifers. It follows that the injection zone is better protected if overlain by number of permeable intervals separated by confining layers.

\section{Acknowledgments}

This project is funded partly by EPA STAR Grant R834384. The author would like to thank S. Aidin Bassam for his input on this paper. The author also thanks J.-P. Nicot, T.A. Meckel, S.A. Hosseini and other colleagues at the Bureau of Economic Geology for their useful comments. The author wishes to thank the three anonymous reviewers for their helpful suggestions. Thank you also to Lana Dieterich who edited the manuscript. Publication authorized by the Director, Bureau of Economic Geology, The University of Texas at Austin.

\section{References}

Abbaszadeh, M. D., and H. Cinco-Ley (1995), Pressure transient behavior in a reservoir with a finite-conductivity fault, SPE Formation Evaluation, 10(1), 26-32. 
Ambastha, A. K., P. G. McLeroy, and A. S. Grader (1989), Effects of a partially communicating fault in a composite reservoir on transient pressure testing, SPE Formation Evaluation, 4(2), 210-218.

Anderson, E. I. (2006), Analytical solutions for flow to a well through a fault, Advances in Water Resources, 29(12), 1790-1803.

Bense, V. F., R. T. Van Balen, and J. J. De Vries (2003), The impact of faults on the hydrogeological conditions in the Roer Valley Rift System: an overview, Neth. J. Geosci./Geol. Mijnbouw, 82, 41-53.

Benson, S. M. and P. Cook (2005), Underground geological storage, in Intergovernmental Panel on Climate Change Special Report on Carbon Dioxide Capture and Storage, coordinating author P. Freund, pp.195-276, Cambridge University Press, Cambridge, UK.

Bixel, H. C., B. K. Larkin, and V. P. H. K. (1963), Effect of linear discontinuities on pressure build-up and drawdown behavior, Journal of Petroleum Technology, 15(8), 885-895.

Buscheck, T. A., Y. Sun, M. Chen, Y. Hao, T. J. Wolery, W. L. Bourcier, B. Court, M. A. Celia, S. Julio Friedmann, and R. D. Aines (2012), Active CO2 reservoir management for carbon storage: Analysis of operational strategies to relieve pressure buildup and improve injectivity, Int. J. Greenhouse Gas Control, 6(0), 230-245.

Caine, J. S., J. P. Evans, and C. B. Forster (1996), Fault zone architecture and permeability structure, Geology, 24(11), 1025-1028.

Cihan, A., Q. Zhou, and J. T. Birkholzer (2011), Analytical solutions for pressure perturbation and fluid leakage through aquitards and wells in multilayered-aquifer systems, Water Resour. Res., 47(10), W10504.

Faulkner, D. R., C. A. L. Jackson, R. J. Lunn, R. W. Schlische, Z. K. Shipton, C. A. J. Wibberley, and M. O. Withjack (2010), A review of recent developments concerning the structure, mechanics and fluid flow properties of fault zones, J. Structural Geology, 32(11), 1557-1575.

Ferris, J. G. (1949), Ground water, in Hydrology, edited by C. O. Wisler and E. F. Brater, pp. 198-272, John Wiley, New York.

Ghaderi, S. M., D. W. Keith, and Y. Leonenko (2009), Feasibility of Injecting Large Volumes of CO2 into Aquifers, Energy Procedia, 1(1), 3113-3120.

Hosseini, S. A., and J.-P. Nicot (2012), Scoping analysis of brine extraction/re-injection for enhanced CO2 storage, Greenhouse Gases: Sci. and Tech.. 2 (3), 172-184.

Jacob, C. E. (1950), Flow of groundwater, in Engineering Hydraulics, edited by H. Rouse, pp. 321-386, John Wiley, New York. 
Jordan, P. D., C. M. Oldenburg, and J.-P. Nicot (2011), Estimating the probability of CO2 plumes encountering faults, Greenhouse Gases: Sci. and Tech., 1(2), 160-174.

Nicot, J.-P. (2008), Evaluation of large-scale CO2 storage on fresh-water sections of aquifers: An example from the Texas Gulf Coast Basin, Int. J. Greenhouse Gas Control, 2(4), 582-593.

Nordbotten, J. M., M. A. Celia, and S. Bachu (2004), Analytical solutions for leakage rates through abandoned wells, Water Resour. Res., 40, W04204.

Raghavan, R. (2010), A composite system with a planar interface, J. Petrol. Sci. and Eng., 70(34), 229-234.

Rahman, N. M. A., M. D. Miller, and L. Mattar (2003 ), Analytical solution to the transient-flow problems for a well located near a finite-conductivity fault in composite reservoirs, in SPE Annual Technical Meeting and Exhibition, SPE Paper 84295, Denver, CO.

Réveillère, A., J. Rohmer, and J.-C. Manceau (2012), Hydraulic barrier design and applicability for managing the risk of CO2 leakage from deep saline aquifers, International Journal of Greenhouse Gas Control, 9(0), 62-71.

Selvadurai, A. P. S. (2012), Fluid leakage through fractures in an impervious caprock embedded between two geologic aquifers, Adv. in Water Resour., 41(0), 76-83.

Shan, C., I. Javandel, and P. A. Witherspoon (1995), Characterization of leaky faults: Study of water flow in aquifer-fault-aquifer systems, Water Resour. Res., 31(12), 2897-2904.

Stauffer, P. H., R. J. Pawar, R. C. Surdam, Z. Jiao, H. Deng, B. C. Lettelier, H. S. Viswanathan, D. L. Sanzo, and G. N. Keating (2011), Application of the CO2 -PENS risk analysis tool to the Rock Springs Uplift, Wyoming, Energy Procedia, 4(0), 4084-4091.

Stehfest, H. (1970), Algorithm368 numerical inversion of Laplace transforms, D-5, Commun. ACM, 13(1), 47-49.

Stewart, G., A. Gupta, and P. Westaway (1984), The interpretation of interference tests in a reservoir with sealing and partially communicating faults, in, SPE European Petroleum Conference, SPE Paper 12967, London, UK.

Sun, A. Y., and J.-P. Nicot (2012), Inversion of pressure anomaly data for detecting leakage at geologic carbon sequestration sites, Adv. in Water Resour., 44(0), 20-29.

Vardoulakis, I., and T. Harnpattanapanich (1986), Numerical Laplace-Fourier transform inversion technique for layered-soil consolidation problems: I. Fundamental solutions and validation, Int. J. Numer. and Analyt. Meth. in Geomech., 10, 347-365. 
Watson, F., S. Mathias, J. van Hunen, S. Daniels, and R. Jones (2012), Dissolution of CO2 from leaking fractures in saline formations, Transp. Porous Media, 1-17.

Wibberley, C. A. J., Yielding, G., and Di Toro, G. (2008), Recent advances in the understanding of fault zone internal structure: A review, in Structure of Fault Zones: Implications for Mechanical and Fluid-flow Properties, edited by C. A. J. Wibberley, W. Kurz, J. Imber, R. E. Holdsworth, C. Collettini, pp. 5-33, Geological Society of London Special Publication 299.

Yaxley, L. M. (1987), Effect of a partially communicating fault on transient pressure behavior, SPE Formation Eval., 2(4), 590-598. 


\section{Appendix A. Solution for two-layer fault system}

Equations (1) through (14) can be written in dimensionless form using dimensionless terms defined by Equations (21) through (23). The governing equations and corresponding boundary and initial conditions are written separately for regions 1 and 2 of the injection zone and the upper zone.

Injection zone (region 1)

$$
\begin{aligned}
& \frac{\partial^{2} P_{D 1}}{\partial x_{D}^{2}}+\frac{\partial^{2} P_{D 1}}{\partial y_{D}^{2}}+\delta\left(x_{D}-1\right) \delta\left(y_{D}\right)=\frac{\partial P_{D 1}}{\partial t_{D}} \\
& P_{D 1}\left(x_{D}, y_{D}, 0\right)=0 \\
& P_{D 1}\left(x_{D}, \pm \infty, t_{D}\right)=0 \\
& P_{D 1}\left(+\infty, y_{D}, t_{D}\right)=0 \\
& \frac{\partial P_{D 1}\left(0, y_{D}, t_{D}\right)}{\partial x_{D}}=\alpha\left(P_{D 1}\left(0, y_{D}, t_{D}\right)-P_{D 2}\left(0, y_{D}, t_{D}\right)\right)+\alpha_{u}\left(P_{D 1}\left(0, y_{D}, t_{D}\right)-P_{D u 1}\left(0, y_{D}, t_{D}\right)\right)
\end{aligned}
$$

Injection zone (region 2)

$$
\begin{aligned}
& \frac{\partial^{2} P_{D 2}}{\partial x_{D}^{2}}+\frac{\partial^{2} P_{D 2}}{\partial y_{D}^{2}}=\frac{\partial P_{D 2}}{\partial t_{D}} \\
& P_{D 2}\left(x_{D}, y_{D}, 0\right)=0 \\
& P_{D 2}\left(x_{D}, \pm \infty, t_{D}\right)=0 \\
& P_{D 2}\left(-\infty, y_{D}, t_{D}\right)=0 \\
& \frac{\partial P_{D 2}\left(0, y_{D}, t_{D}\right)}{\partial x_{D}}+\alpha_{u}\left(P_{D 2}\left(0, y_{D}, t_{D}\right)-P_{D u 2}\left(0, y_{D}, t_{D}\right)\right)=\alpha\left(P_{D 1}\left(0, y_{D}, t_{D}\right)-P_{D 2}\left(0, y_{D}, t_{D}\right)\right)
\end{aligned}
$$

Upper zone (region 1) 


$$
\begin{aligned}
& \frac{\partial^{2} P_{D u 1}}{\partial x_{D}^{2}}+\frac{\partial^{2} P_{D u 1}}{\partial y_{D}^{2}}=\frac{1}{\eta_{D}} \frac{\partial P_{D u 1}}{\partial t_{D}} \\
& P_{D u 1}\left(x_{D}, y_{D}, 0\right)=0 \\
& P_{D u 1}\left(x_{D}, \pm \infty, t_{D}\right)=0 \\
& P_{D u 1}\left(+\infty, y_{D}, t_{D}\right)=0 \\
& \alpha_{u}\left(P_{D 1}\left(0, y_{D}, t_{D}\right)-P_{D u 1}\left(0, y_{D}, t_{D}\right)\right)+T_{D} \frac{\partial P_{D u 1}\left(0, y_{D}, t_{D}\right)}{\partial x_{D}}=\alpha h_{D}\left(P_{D u 1}\left(0, y_{D}, t_{D}\right)-P_{D u 2}\left(0, y_{D}, t_{D}\right)\right)
\end{aligned}
$$

Upper zone (region 2)

$$
\begin{aligned}
& \frac{\partial^{2} P_{D u 2}}{\partial x_{D}^{2}}+\frac{\partial^{2} P_{D u 2}}{\partial y_{D}^{2}}=\frac{1}{\eta_{D}} \frac{\partial P_{D u 2}}{\partial t_{D}} \\
& P_{D u 2}\left(x_{D}, y_{D}, 0\right)=0 \\
& P_{D u 2}\left(x_{D}, \pm \infty, t_{D}\right)=0 \\
& P_{D u 2}\left(-\infty, y_{D}, t_{D}\right)=0 \\
& \alpha_{u}\left(P_{D 2}\left(0, y_{D}, t_{D}\right)-P_{D u 2}\left(0, y_{D}, t_{D}\right)\right)+\alpha h_{D}\left(P_{D u 1}\left(0, y_{D}, t_{D}\right)-P_{D u 2}\left(0, y_{D}, t_{D}\right)\right)=T_{D} \frac{\partial P_{D u 2}\left(0, y_{D}, t_{D}\right)}{\partial x_{D}}
\end{aligned}
$$

Taking the sequential Laplace and (exponential) Fourier transform as defined by Equations (15) and (16) results in the following set of equations:

Injection zone (region 1):

$$
\begin{aligned}
& \frac{d^{2} \overline{\bar{P}}_{D 1}}{d x_{D}^{2}}-A^{2} \overline{\bar{P}}_{D 1}=-\frac{1}{s} \delta\left(x_{D}-1\right) \\
& \overline{\bar{P}}_{D 1}(+\infty)=0 \\
& \frac{d \overline{\bar{P}}_{D 1}(0)}{d x_{D}}=\alpha\left(\overline{\bar{P}}_{D 1}(0)-\overline{\bar{P}}_{D 2}(0)\right)+\alpha_{u}\left(\overline{\bar{P}}_{D 1}(0)-\overline{\bar{P}}_{D u 1}(0)\right)
\end{aligned}
$$


where $A^{2}=s+\omega^{2}$.

Injection zone (region 2):

$\frac{d^{2} \overline{\bar{P}}_{D 2}}{d x_{D}^{2}}-A^{2} \overline{\bar{P}}_{D 2}=0$

$\overline{\bar{P}}_{D 2}(-\infty)=0$

$\frac{d \overline{\bar{P}}_{D 2}(0)}{d x_{D}}+\alpha_{u}\left(\overline{\bar{P}}_{D 2}(0)-\overline{\bar{P}}_{D u 2}(0)\right)=\alpha\left(\overline{\bar{P}}_{D 1}(0)-\overline{\bar{P}}_{D 2}(0)\right)$

Upper zone (region 1):

$$
\begin{aligned}
& \frac{d^{2} \overline{\bar{P}}_{D u 1}}{d x_{D}^{2}}-A_{u}^{2} \overline{\bar{P}}_{D u 1}=0 \\
& \overline{\bar{P}}_{D u 1}(+\infty)=0 \\
& \alpha_{u}\left(\overline{\bar{P}}_{D 1}(0)-\overline{\bar{P}}_{D u 1}(0)\right)+T_{D} \frac{d \overline{\bar{P}}_{D u 1}(0)}{d x_{D}}=\alpha h_{D}\left(\overline{\bar{P}}_{D u 1}(0)-\overline{\bar{P}}_{D u 2}(0)\right)
\end{aligned}
$$

where $A_{u}^{2}=\omega^{2}+\frac{S}{\eta_{D}}$.

Upper zone (region 2):

$$
\begin{aligned}
& \frac{d^{2} \overline{\bar{P}}_{D u 2}}{d x_{D}^{2}}-A_{u}^{2} \overline{\bar{P}}_{D u 2}=0 \\
& \overline{\bar{P}}_{D u 2}(-\infty)=0 \\
& \alpha_{u}\left(\overline{\bar{P}}_{D 2}(0)-\overline{\bar{P}}_{D u 2}(0)\right)+\alpha h_{D}\left(\overline{\bar{P}}_{D u 1}(0)-\overline{\bar{P}}_{D u 2}(0)\right)=T_{D} \frac{d \overline{\bar{P}}_{D u 2}(0)}{d x_{D}}
\end{aligned}
$$

Solution of the above system of ODE's considering the corresponding boundary conditions is given by: 


$$
\begin{aligned}
& \overline{\bar{P}}_{D 1}=\frac{1}{2 s A}\left(e^{-\left|x_{D}-1\right| A}\right)+C_{1} e^{-A x_{D}} \\
& \overline{\bar{P}}_{D 2}=C_{2} e^{A x_{D}} \\
& \overline{\bar{P}}_{D u 1}=C_{u 1} e^{-A_{u} x_{D}} \\
& \overline{\bar{P}}_{D u 2}=C_{u 2} e^{A_{u} x_{D}}
\end{aligned}
$$

The coefficients $\left(C_{1}, C_{2}, C_{u 1}, C_{u 2}\right)$ are obtained by solving the following system of four equations and four unknowns given by:

$$
\begin{aligned}
& C_{2} A+\alpha_{u}\left(C_{2}-C_{u 2}\right)=\alpha\left(C_{0}+C_{1}-C_{2}\right) \\
& \left(C_{0}-C_{1}\right) A=\alpha\left(C_{0}+C_{1}-C_{2}\right)+\alpha_{u}\left(C_{0}+C_{1}-C_{u 1}\right) \\
& \alpha_{u}\left(C_{0}+C_{1}-C_{u 1}\right)-T_{D} C_{u 1} A_{u}=\alpha h_{D}\left(C_{u 1}-C_{u 2}\right) \\
& \alpha_{u}\left(C_{2}-C_{u 2}\right)+\alpha h_{D}\left(C_{u 1}-C_{u 2}\right)=T_{D} C_{u 2} A_{u}
\end{aligned}
$$

where $C_{0}=\frac{1}{2 s A} e^{-A}$.

To obtain the solution, we can numerically evaluate the coefficients during the Laplace-Fourier inversion. However, we can also obtain the coefficients explicitly, which results in a closed-form solution given by:

$$
\begin{aligned}
& \bar{P}_{D 1}=\frac{1}{2 s A}\left(e^{-A\left|x_{D}-1\right|}+\frac{A^{2}\left(\alpha_{u}+A_{u} T_{D}\right)\left(\alpha_{u}+A_{u} T_{D}+2 \alpha h_{D}\right)-2 \alpha \alpha_{u} A_{u} T_{D}\left(\alpha_{u}+2 \alpha h_{D}+\alpha_{u} h_{D}+A_{u} T_{D}\right)-\alpha_{u} A_{u} T_{D} \alpha_{u}}{\left(A a_{u}+A A_{u} T_{D}+a_{u} A_{u} T_{D}\right)\left(2 a a_{u}+A a_{u}+4 a^{2} h_{D}+2 a A h_{D}+2 a a_{u} h_{D}+2 a A_{u} T_{D}+A A_{u} T_{D}+a_{u} A_{u} T_{D}\right)} e^{-A\left(x_{D}+1\right)}\right) \\
& \bar{P}_{D 2}=\frac{\alpha}{s}\left(\frac{\alpha_{u}\left(\alpha_{u}+2 \alpha h_{D}+\alpha_{u} h_{D}\right)+2 A_{u} T_{D}\left(\alpha_{u}+\alpha h_{D}\right)+A_{u}^{2} T_{D}^{2}}{\left(A a_{u}+A A_{u} T_{D}+a_{u} A_{u} T_{D}\right)\left(2 a a_{u}+A a_{u}+4 a^{2} h_{D}+2 a A h_{D}+2 a a_{u} h_{D}+2 a A_{u} T_{D}+A A_{u} T_{D}+a_{u} A_{u} T_{D}\right)} e^{A\left(x_{D}-1\right)}\right) \\
& \overline{\bar{P}}_{D u 1}=\frac{\alpha_{u}}{S}\left(\frac{\alpha \alpha_{u}+A \alpha_{u}+2 \alpha^{2} h_{D}+\alpha A h_{D}+\alpha \alpha_{u} h_{D}+\alpha A_{u} T_{D}+A A_{u} T_{D}+\alpha_{u} A_{u} T_{D}}{\left(A a_{u}+A A_{u} T_{D}+a_{u} A_{u} T_{D}\right)\left(2 a a_{u}+A a_{u}+4 a^{2} h_{D}+2 a A h_{D}+2 a a_{u} h_{D}+2 a A_{u} T_{D}+A A_{u} T_{D}+a_{u} A_{u} T_{D}\right)} e^{-A_{u} x_{D}-A}\right)
\end{aligned}
$$


$\overline{\bar{P}}_{\text {Du2 }}=\frac{\alpha \alpha_{u}}{s}\left(\frac{\alpha_{u}+2 \alpha h_{D}+A h_{D}+\alpha_{u} h_{D}+A_{u} T_{D}}{\left(A a_{u}+A A_{u} T_{D}+a_{u} A_{u} T_{D}\right)\left(2 a a_{u}+A a_{u}+4 a^{2} h_{D}+2 a A h_{D}+2 a a_{u} h_{D}+2 a A_{u} T_{D}+A A_{u} T_{D}+a_{u} A_{u} T_{D}\right)} e^{A_{u} x_{D}-A}\right)$

The arithmetic mean of the pressure change at the fault can be evaluated based on the pressure changes given in Equations (A41)-(A44) considering $x_{D}=0$. As a result, the mean pressure change for the injection and upper zones are obtained by:

$$
\begin{aligned}
& \overline{\bar{P}}_{D, \text { mean }}\left(x_{D}=0\right)=\frac{\overline{\bar{P}}_{D 1}\left(x_{D}=0\right)+\overline{\bar{P}}_{D 2}\left(x_{D}=0\right)}{2}=\frac{\mathrm{e}^{-A}}{2 s} \frac{\alpha_{u}+A_{u} T_{D}}{A \alpha_{u}+A A_{u} T_{D}+\alpha_{u} A_{u} T_{D}} \\
& \overline{\bar{P}}_{\text {Du,mean }}\left(x_{D}=0\right)=\frac{\overline{\bar{P}}_{\text {Du1 }}\left(x_{D}=0\right)+\overline{\bar{P}}_{\text {Du2 }\left(x_{D}=0\right)}}{2}=\frac{\mathrm{e}^{-A}}{2 s} \frac{\alpha_{u}}{A a_{u}+A A_{u} T_{D}+\alpha_{u} A_{u} T_{D}}
\end{aligned}
$$

Based on Equations (A45) and (A46) the arithmetic mean of the pressure at the fault plane is independent of parameters $\alpha$ and $h_{D}$. 


\section{Appendix B. Fourier-Laplace transforms inversion}

The Fourier transform can be inverted through discretized integration [Vardoulakis and Harnpattanapanich, 1986]. The Inverse Fourier transform is defined by:

$\bar{P}_{D}\left(x_{D}, y_{D}, s\right)=\mathcal{F}^{-1}\left\{\overline{\bar{P}}_{D}\left(x_{D}, \omega, s\right)\right\}=\frac{1}{2 \pi} \int_{-\infty}^{+\infty} \overline{\bar{P}}_{D}(\omega) e^{-i \omega y_{D}} d \omega$

There is $\omega_{0}$ beyond which $\overline{\bar{P}}_{D}$ does not vary significantly. Then Equation (B1) can be approximated by:

$\bar{P}_{D}=\frac{1}{2 \pi} \int_{-\omega_{0}}^{\omega_{0}} \overline{\bar{P}}_{D}(\omega) e^{-i \omega y_{D}} d \omega$

Considering $\Delta \omega=2 \omega_{0} / N$, Equation (B2) can be written in the form of series approximation:

$\bar{P}_{D}=\frac{\Delta \omega}{2 \pi} \sum_{n=-N / 2}^{N / 2} \overline{\bar{P}}_{D}(n \Delta \omega) e^{-i y_{D} n \Delta \omega}$

Next, the inverse Laplace transform of $\bar{P}_{D}$ is calculated using the Stehfest [1970] algorithm, and the solution is obtained in the time-space domain.

If $\overline{\bar{P}}_{D}$ is an even function of $\omega$ (as is the case for pressure changes in the injection and upper zones), we can further simplify the Fourier inversion:

$\bar{P}_{D}=\frac{\Delta \omega}{\pi} \sum_{n=0}^{N / 2} \overline{\bar{P}}_{D}(n \Delta \omega) e^{-i y_{D} n \Delta \omega}$

Defining $\Delta \omega=\omega_{0} / N$ we get $\bar{P}_{D}=\frac{\Delta \omega}{\pi} \sum_{n=0}^{N} \overline{\bar{P}}_{D}(n \Delta \omega) e^{-i y_{D} n \Delta \omega}$.

The above inversion method of Fourier transform is computationally expensive. A better approach is to use the Inverse Fast Fourier Transform method, which utilizes an algorithm that does the same thing as the above method but in much less time. The Fast Fourier Transform (fft) function defined in MATLAB corresponds to: 
$\bar{P}\left(y_{D}\right)=\int_{-\infty}^{\infty} \overline{\bar{P}}(\omega) e^{-i y_{D} \omega} d \omega$

This is $2 \pi$ times the inverse Fourier transform defined in this paper. Because the function to be inverted is even and symmetric, we should sample a portion of the signal in $\omega$ while keeping the symmetric behavior. Otherwise, the space-domain response will not be real anymore. The function vanishes beyond a certain $\omega$. Therefore, it is not required to take large values of $\omega$ in the calculation. However, the region over which the function varies should be sampled at high resolution. Such a region is given by the number of samples divided by sampling frequency. Scaling is required because fft function assumes that the sampling rate $\left(d y_{D}\right)$ is 1 . Thus, in order to consider the actual sampling frequency, we need to divide the output of the fft by the sampling frequency. Next, the results should be shifted (fftshift function in MATLAB) so that the zerofrequency component is at the center. 


\section{Appendix C. Multilayer system}

For regions 1 and 2 of the injection zone, Equations (1) through (5) remain the same. Equations (6) and (7) are replaced by:

$$
\begin{aligned}
& \Delta P_{1}(0, y, t)=\Delta P_{2}(0, y, t) \\
& \frac{k h}{\mu} \frac{\partial \Delta P_{1}(0, y, t)}{\partial x}=\frac{k h}{\mu} \frac{\partial \Delta P_{2}(0, y, t)}{\partial x}+\frac{k_{f v, 1} w_{f}}{\mu} \frac{\Delta P_{1}(0, y, t)-\Delta P_{u 2,1}(0, y, t)}{L_{1}}
\end{aligned}
$$

Neglecting the lateral resistance to flow of the fault plane, the pressure change at both regions 1 and 2 of the upper zones $(i=1,2, \ldots, N)$ are the same. We write the equations for region 2 for convenience:

$\frac{\partial^{2} \Delta P_{u 2, i}}{\partial x^{2}}+\frac{\partial^{2} \Delta P_{u 2, i}}{\partial y^{2}}=\frac{1}{\eta_{u i}} \frac{\partial \Delta P_{u 2, i}}{\partial t}$

$\Delta P_{u 2, i}(x, y, 0)=0$

$\Delta P_{u 2, i}(x, \pm \infty, t)=0$

$\Delta P_{u 2, i}(-\infty, y, t)=0$

$\frac{k_{f v, i} w_{f}}{\mu} \frac{\Delta P_{u 2, i-1}(0, y, t)-\Delta P_{u 2, i}(0, y, t)}{L_{i}}=2 \sum_{j=i}^{N} \frac{k_{u j} h_{u j}}{\mu} \frac{\partial \Delta P_{u 2, j}(0, y, t)}{\partial x}$

where $N$ is the number of aquifers intersected by the leaky fault and $\Delta P_{u 2,0}=\Delta P_{1}$. Making the equations dimensionless based on Equations (21) through (23) followed by Laplace and Fourier transforms, we end up with the following system:

$\underline{\text { Region } 1 \text { of injection zone: }}$

$\frac{d^{2} \overline{\bar{P}}_{D 1}}{d x_{D}^{2}}-A^{2} \overline{\bar{P}}_{D 1}=-\frac{1}{S} \delta\left(x_{D}-1\right)$

$\overline{\bar{P}}_{D 1}(+\infty)=0$ 
$\overline{\bar{P}}_{D 1}(0)=\overline{\bar{P}}_{D 2}(0)$

where $A^{2}=s+\omega^{2}$.

Region 2 of injection zone:

$\frac{d^{2} \overline{\bar{P}}_{D 2}}{d x_{D}^{2}}-A^{2} \overline{\bar{P}}_{D 2}=0$

$\overline{\bar{P}}_{D 2(-\infty)}=0$

$\frac{d \overline{\bar{P}}_{D 1}(0)}{d x_{D}}=\frac{d \overline{\bar{P}}_{D 2}(0)}{d x_{D}}+2 \alpha_{u 1}\left(\overline{\bar{P}}_{D 1}(0)-\overline{\bar{P}}_{D u 2,1}(0)\right)$

Upper zones $(i=1,2, \ldots, N)$ :

$\frac{d^{2} \overline{\bar{P}}_{D u 2, i}}{d x_{D}^{2}}-A_{u i}^{2} \overline{\bar{P}}_{D u 2, i}=0$

$\overline{\bar{P}}_{D u 2, i}(-\infty)=0$

$\alpha_{u i}\left(\overline{\bar{P}}_{D u 2, i-1}(0)-\overline{\bar{P}}_{D u 2, i}(0)\right)=\sum_{j=i}^{N} T_{D j} \frac{d \overline{\bar{P}}_{D u 2, j}(0)}{d x_{D}}$

where: $A_{u i}^{2}=\omega^{2}+\frac{s}{\eta_{D i}}$.

The solution for the above system of ODE's is given by:

$$
\begin{aligned}
& \overline{\bar{P}}_{D 1}=\frac{1}{2 s A}\left(e^{-\left|x_{D}-1\right| A}\right)+C_{1} e^{-A x_{D}} \\
& \overline{\bar{P}}_{D 2}=C_{2} e^{A x_{D}} \\
& \overline{\bar{P}}_{D u 2, i}=C_{u i} e^{A_{u} x_{D}} \text { for } i=1,2, \ldots, N
\end{aligned}
$$

where the coefficients $C_{1}, C_{2}$, and $C_{u i}$ (for $i=1,2, \ldots, N$ ) are obtained by solving the following system of equations: 
$C_{0}+C_{1}=C_{2}$

$\left(C_{0}-C_{1}\right) A=C_{2} A+2 \alpha_{u 1}\left(C_{0}+C_{1}-C_{u 1}\right)$

$\alpha_{u i}\left(C_{u, i-1}-C_{u i}\right)=\sum_{j=i}^{N} T_{D j} A_{u j} C_{u j}$ for $i=1,2, \ldots, N$

For the above system we have $C_{u, 0}=C_{0}+C_{1}$, and $C_{0}=\frac{1}{2 s A} e^{-A}$.

The leakage rate is then calculated by:

$q_{l, i}=2 \frac{k_{u i} h_{u i}}{\mu} \int_{y=-\infty}^{\infty} \frac{\partial \Delta P_{u 2, i}(0, y, t)}{\partial x} d y$

and in dimensionless form:

$q_{l D, i}=2 T_{D i} \int_{-\infty}^{\infty} \frac{\partial P_{D u 2, i}\left(0, y_{D}, t_{D}\right)}{\partial x_{D}} d y_{D}$

where

$\left.\frac{d \overline{\bar{P}}_{D u 2, i}}{d x_{D}}\right|_{x_{D}=0}=C_{u i} A_{u i}$ 


\section{Appendix D. Derivation of two-layer leakage rate from multilayer solution}

For the two-layer system $N=1$ and therefore, Equation (29) results in a single equation which provides the leakage rate to the upper layer. For convenience, we re-write Equation (29) excluding the index 1 (which represents the upper layer\#1) and bring the coefficient 2 into the integral:

$q_{l D}=T_{D} \int_{-\infty}^{\infty} 2 \frac{\partial P_{D u 2}\left(0, y_{D}, t_{D}\right)}{\partial x_{D}} d y_{D}$

For the multilayer solution to reduce to the two-layer solution, the integrand of Equation (D1) in Laplace-Fourier domain must be equal to that given by Equation (28). Based on Equation (31) the integrand of Equation (D1) in Laplace-Fourier domain is obtained by:

$$
\left.2 \frac{d \overline{\bar{P}}_{\text {Du2 }}}{d x_{D}}\right|_{x_{D}=0}=2 C_{u} A_{u}
$$

The coefficient $C_{u}$ is obtained by solving Equation (32) written for two-layer system:

$$
\left[\begin{array}{lll}
-1 & 1 & 0 \\
A & A+2 \alpha_{u} & -2 \alpha_{u} \\
0 & -\alpha_{u} & \alpha_{u}+T_{D} A_{u}
\end{array}\right]\left[\begin{array}{l}
C_{1} \\
C_{2} \\
C_{u}
\end{array}\right]=\frac{e^{-A}}{2 s A}\left[\begin{array}{l}
1 \\
A \\
0
\end{array}\right]
$$

By solving the above linear system of 3 equations and 3 unknowns we get:

$$
C_{u}=\frac{\alpha_{u}}{\alpha_{u} A_{u} T_{D}+A \alpha_{u}+A A_{u} T_{D}} \frac{e^{-A}}{2 s}
$$

Combining Equations (D2) and (D4) gives:

$\left.2 \frac{d \overline{\bar{P}}_{D u 2}}{d x_{D}}\right|_{x_{D}=0}=\frac{A_{u} \alpha_{u}}{A \alpha_{u}+A A_{u} T_{D}+\alpha_{u} A_{u} T_{D}} \frac{e^{-A}}{s}$

Right hand sides of equations (D5) and (28) are identical and so are the leakage rates. 
Figure 1. Schematic of a typical fault system. Vertical inter-formational flow may be accommodated by the damage zone surrounding the fault core in response to pressure perturbations caused by injection/production.

Figure 2. Schematic of the idealized fault-reservoir system modeled in this paper. The fault separates region 1 from region 2 .

Figure 3. Zoomed in fault plane where (a) the fault core and damage zones are lumped and assigned a single distinct vertical permeability and (b) the damage zones are assigned the same vertical permeabilities that are horizontally in communication, given the fault's horizontal permeability. Using the former approach for modeling the vertical flow at the fault plane leads to equal leakage rates to regions 1 and $2\left(q_{11}=q_{12}\right)$, because it assumes that the damage zones are in perfect communication. However, the latter approach leads to $q_{l 1} \neq q_{l 2}$. The latter approach is more consistent with the general architecture of the fault, in which the fault core is surrounded by damage zones and is used in this paper.

Figure 4. Schematic of a fault intersecting multilayer system. The fault separates region 1 from region 2.

Figure 5. Verification of the analytical solution presented here against the Shan et al. [1995] solution, considering $x_{D}=0.5, T_{D}=1$, and $\alpha_{u}=0.02$ for various values of $\alpha$.

Figure 6. Dimensionless leakage rate to the upper aquifer using our solution compared with the results presented in Shan et al. [1995], considering zero pressure change in the upper aquifer.

Figure 7. Comparison of pressure change in the target aquifer using the analytical solution presented here against the Shan et al. [1995] solution, considering various values of $\alpha$ for $T_{D} \rightarrow \infty$.

Figure 8. Pressure change in (a) the target aquifer and (b) the upper aquifer, considering $T_{D}=1$ for various values of $\alpha$.

Figure 9. Pressure change in (a) the target aquifer and (b) the upper aquifer, considering $T_{D}=1$ and $\alpha=1$ for various values of $h_{D}$.

Figure 10. Dimensionless leakage rate (solid line) and dimensionless leakage volume (dashed line) to the top, middle, and bottom aquifers overlying the injection zone versus time for injection in (a) log-log and (b) semi-log forms. Red curves correspond to the equivalent twolayer system. 
Figure 11. Dimensionless leakage volume to overlying formation(s) of multilayer system example (black curves) and equivalent two-layer system (red curves) after 1, 5, and 25 years of injection. 


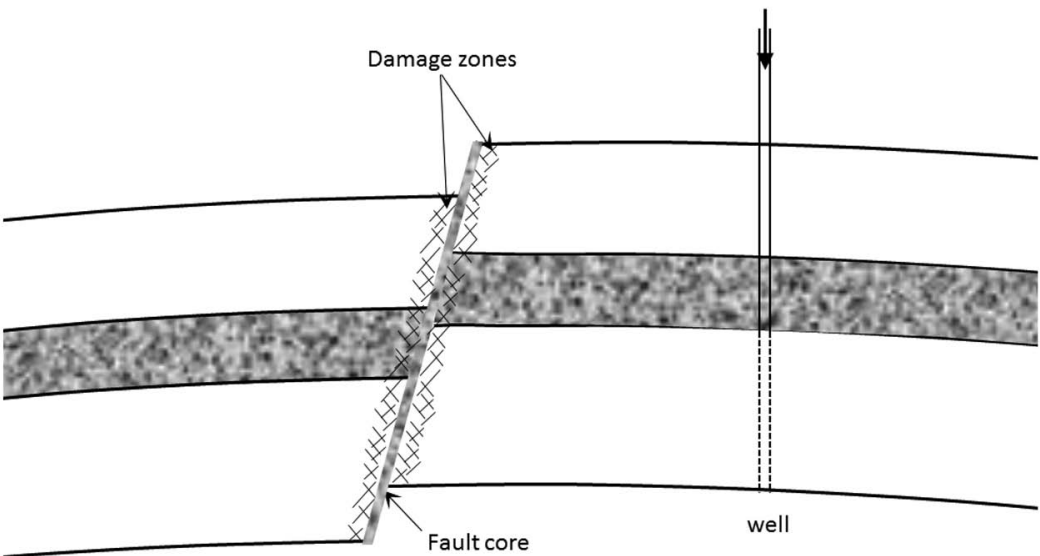




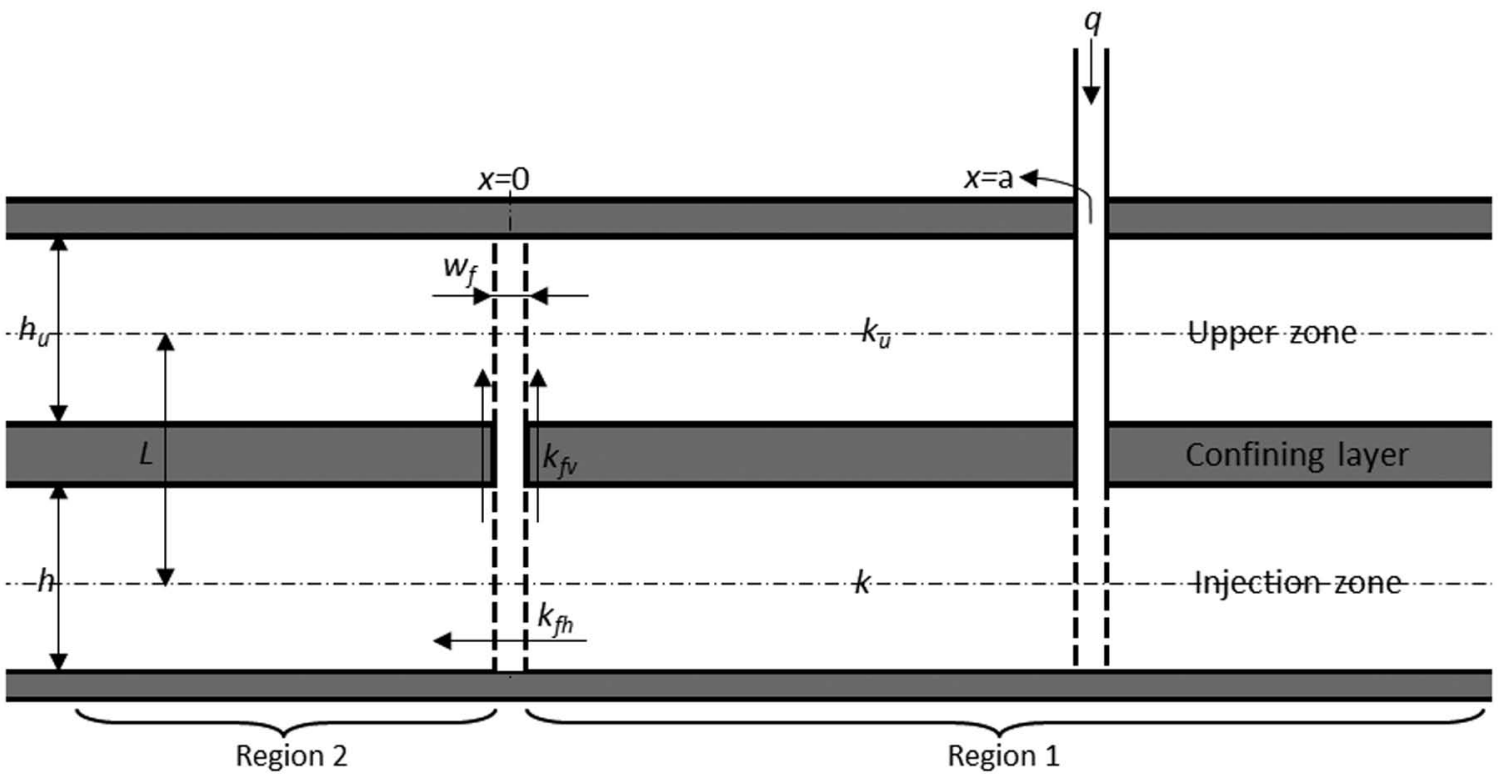


(a)

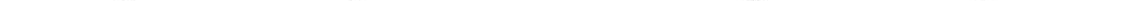

(b) 


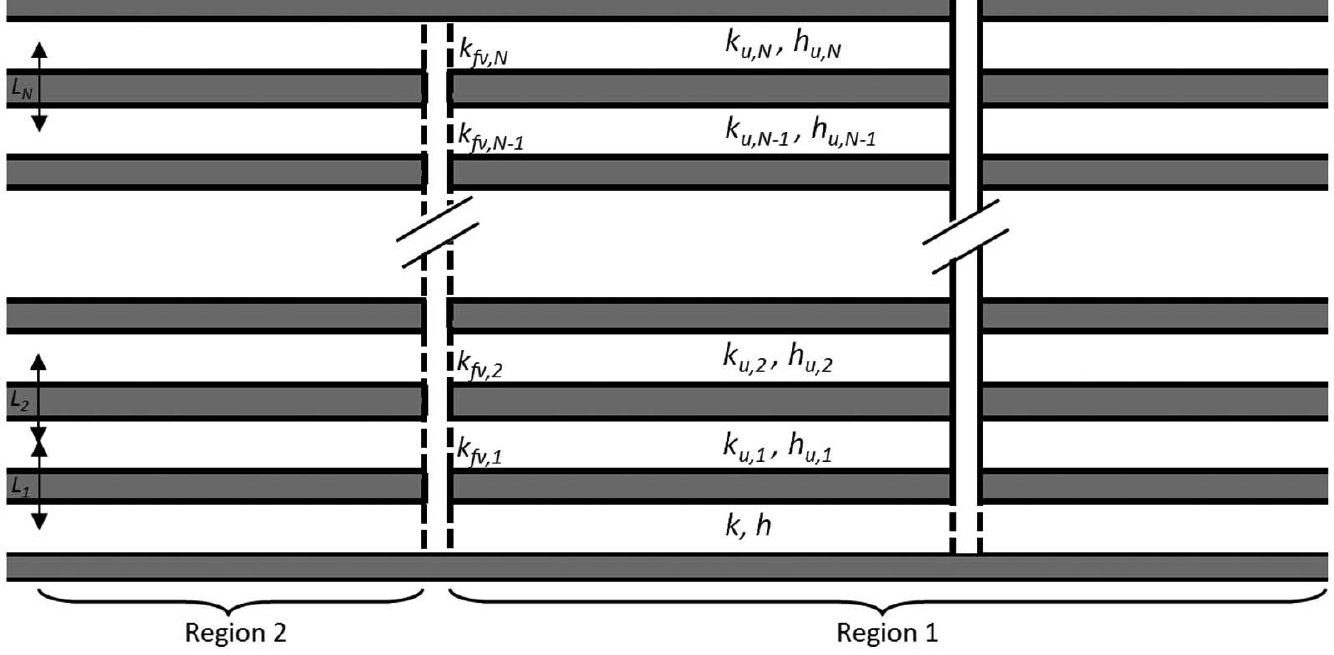




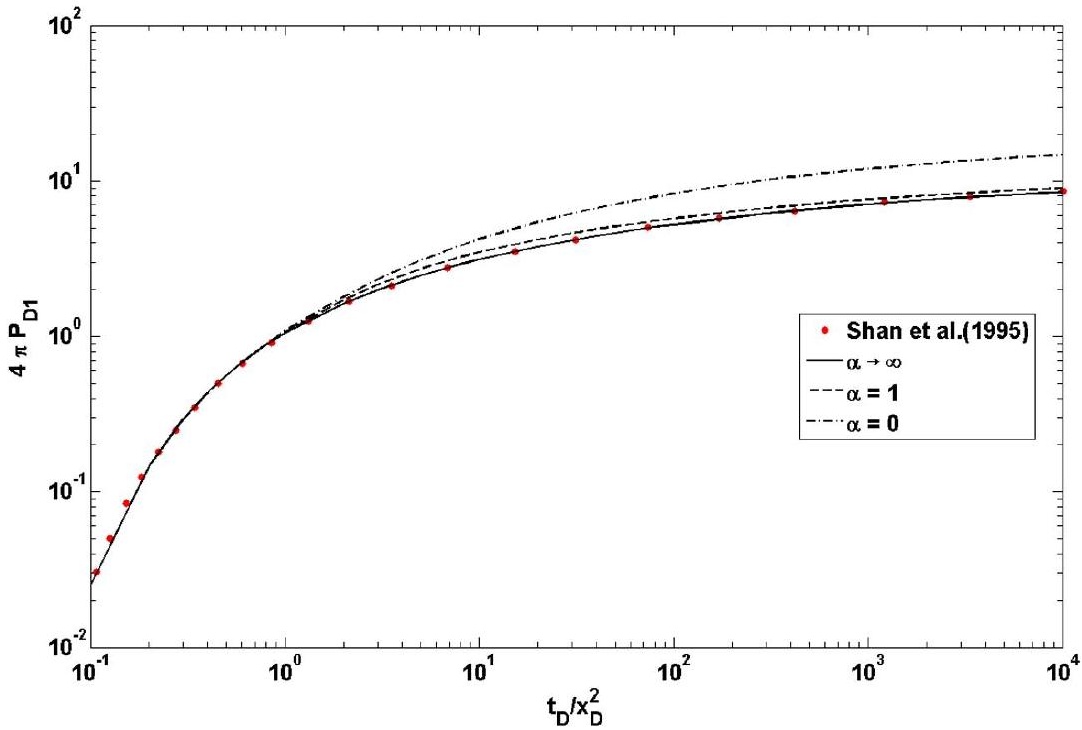




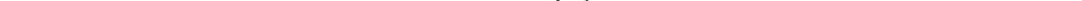


(a)

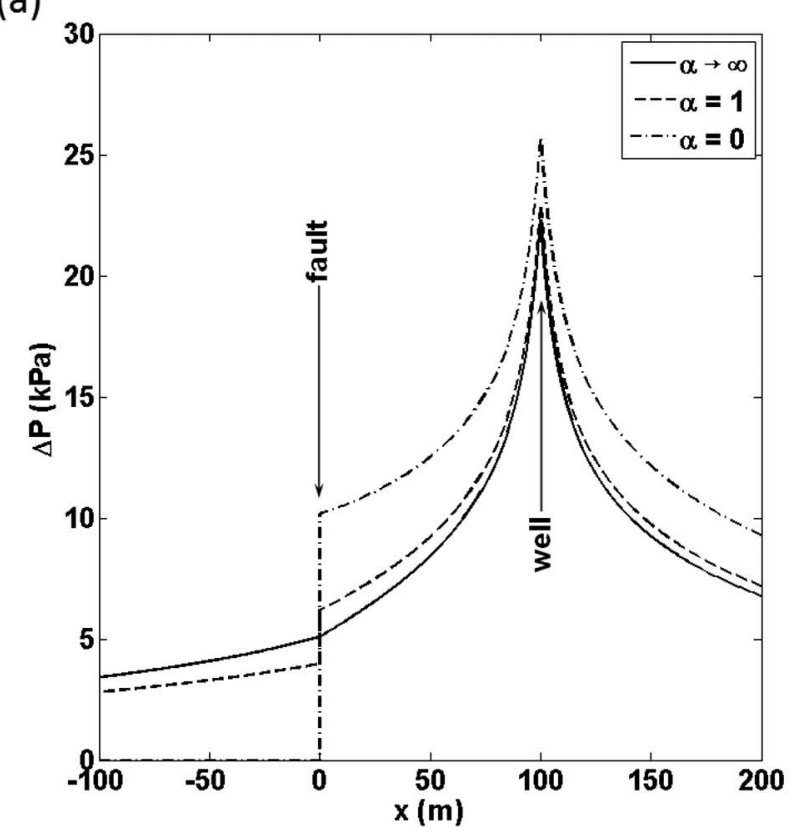

(b)

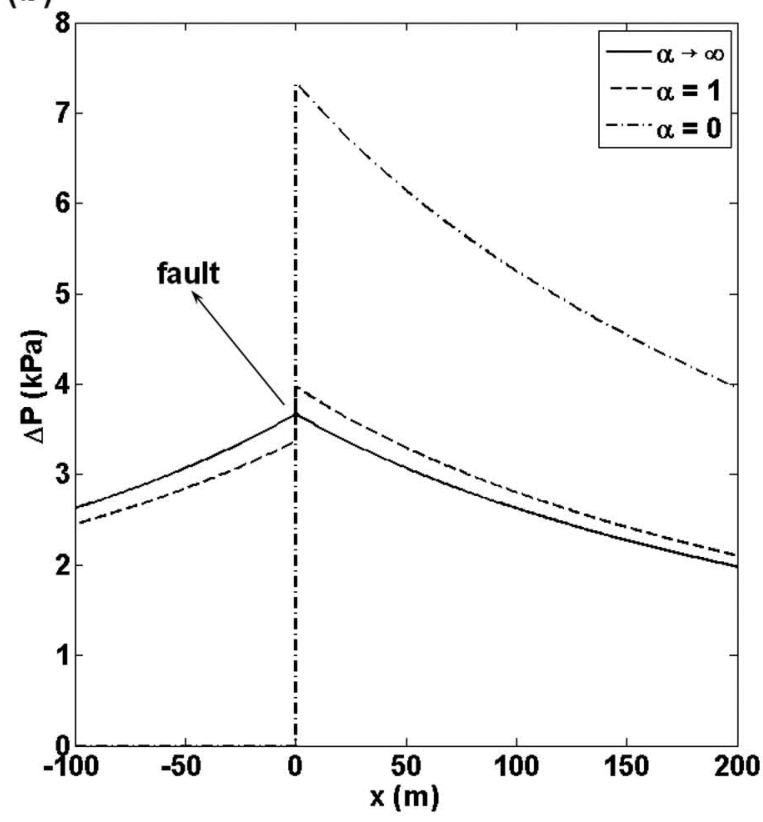


(a)

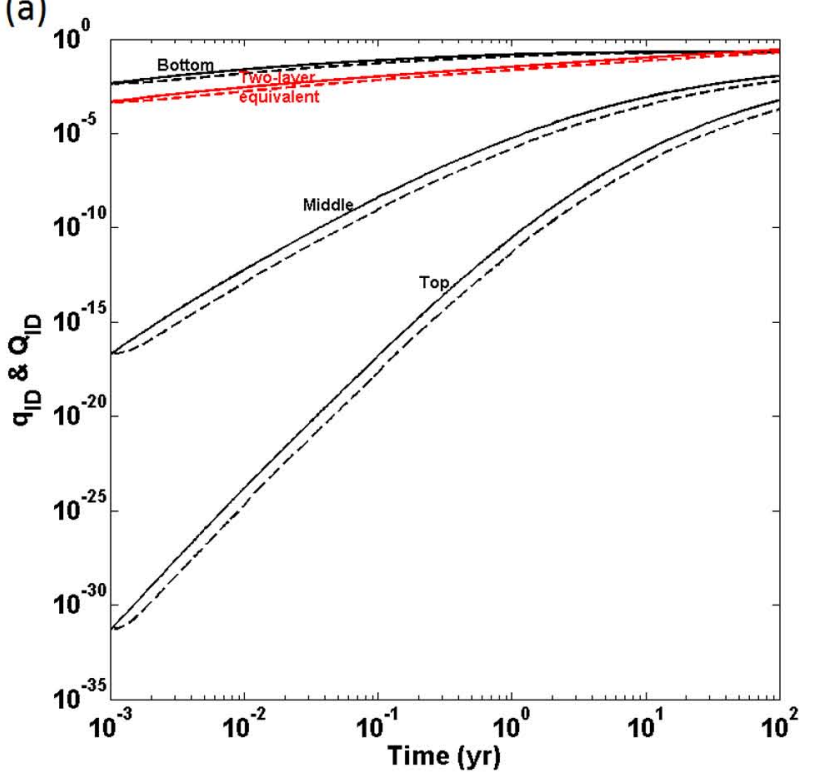

(b)

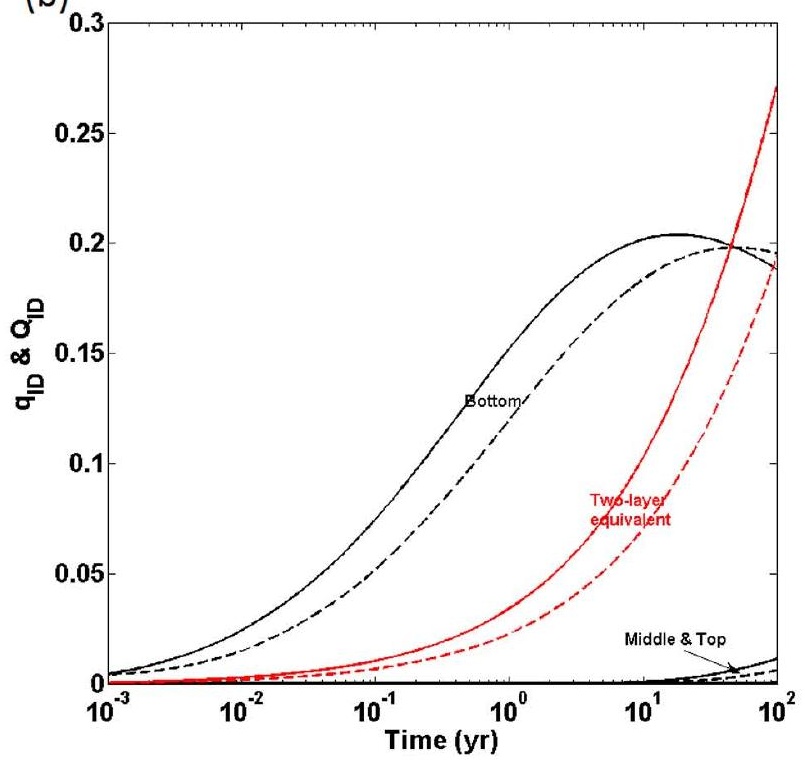




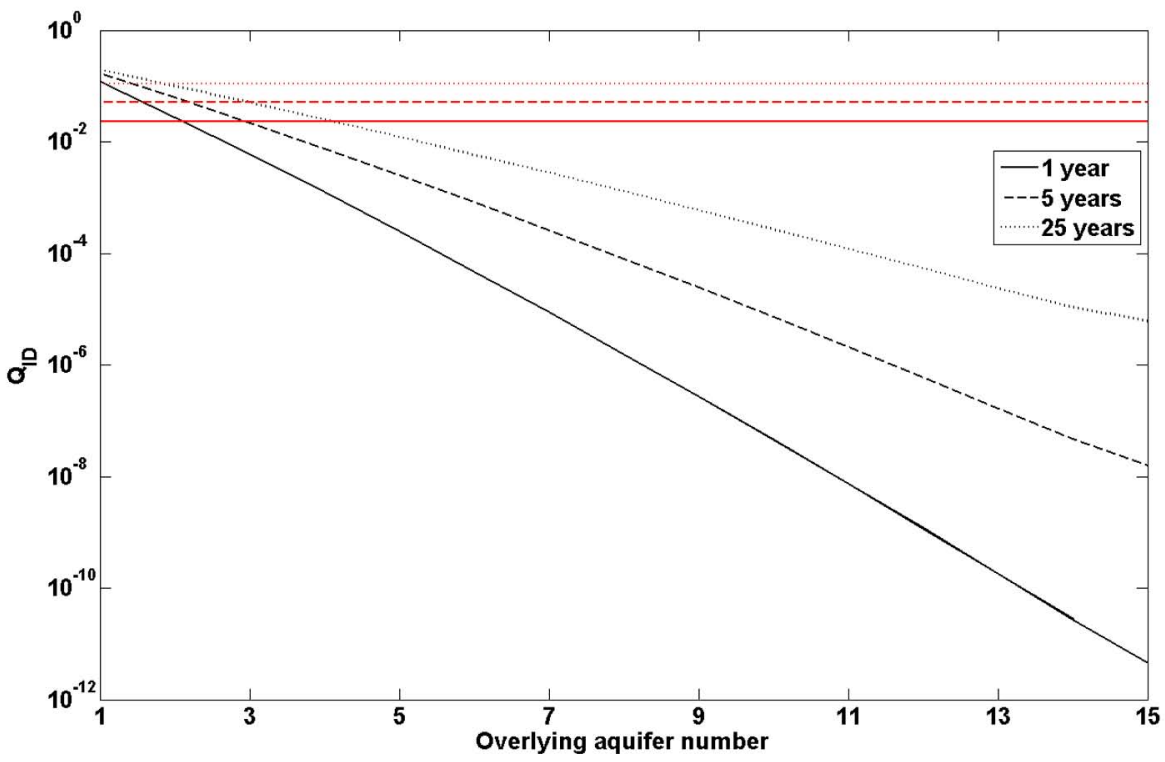

